\title{
Chitosan capped Silver nanoparticles used as Pressure sensors
}

\author{
K. Praveenkumar*a, M. K. Rabinal ${ }^{\mathrm{b}}$, M. N. Kalasad ${ }^{\mathrm{b}}$, T. Sankarappa ${ }^{\mathrm{a}}$, \\ Mahesh D. Bedre \\ ${ }^{a}$ Department of Physics, Gulbarga University, Gulbarga-585106, Karnataka State, India. \\ ${ }^{b}$ Department of Physics, Karnatak University, Dharwad-580003, Karnataka State, India. \\ ${ }^{c}$ Mahesh P.U.College of Science, Gulbarga-585102, Karnataka State, India. \\ Corresponding author: praveenkphysics@gmail.com
}

\begin{abstract}
In the present work, we report the synthesis and characterization of silver nanoparticles, capped with chitosan (biopolymer). The majority of the particles produced in this way had sizes around 18 nm. Composite films of capped silver nanoparticles and chitosan polymer were studied to understand the charge transport under different pressure. Films of different compositions were prepared to measure current voltage curves across the film thickness. The results reveal that these materials exhibit electrical conductivity as predicted by the "classical theory of percolation". Pressure dependent electrical conductivity and these composites can be explored to develop low cost pressure sensors.
\end{abstract}

Keywords: Chitosan - silver nanoparticles, UV-Vis. Spectroscopy, X-ray diffraction, FTIR spectroscopy, SEM, Electrical conductivity, pressure sensors.

\section{Introduction}

The future of sustainable nanoscale science and technology not only depends on overcoming knowledge barriers in developing newer principles, but also on appropriately addressing the environmental and societal concerns, specially when largescale material production is involved. The primary challenges in this regard are the maximization of sage of environmentally friendly materials in the generation of nanoscale based products. Among the many products that have been developed, polymer-metal nanoparticle composites seem to receive special attention because of their application potential in sensors [1,2], actuators [3], electrodes for fuel cells [4], supported micro-heterogeneous and heterogeneous catalyses $[5,6,7,8]$ and in optical and optoelectronic devices $[9,10,11]$

Chitosan, a novel biopolymer, is a product of deacetylation of chitin, which is the second most abundant natural polymer in the world after cellulose. The biocompatibility and antibacterial properties of chitosan and its being an environmentally friendly polyelectrolyte makes it appealing for several applications such as water treatment [12], novel fibers for textiles [13], photographic paper [14,15], biodegradable films [16,17], biomedical devices and microcapsule implants for controlled release in drug delivery $[18,19,20]$

In recent years polymers have attracted considerable attention due to their importance in emerging technologies [21,22,23,24,25]. These materials have been widely studied for their optoelectronic and electrochemical properties [26]. It is well known that the electrical properties of composites depend on concentration, size and shape of conductive particles. Further, the intermixing of two phases will also play a major role in controlling physical properties [27,28,29]. There is a scientific and technical interest to study the physical properties of composites consisting of conductive particles and rubber (compressible medium) as an insulating matrix. The high compressibility of latter permits to control interparticle separation between conducting aggregates by applied pressure. This makes it possible to tune the system from quantum (tunneling) to classical (percolation) regimes by applied pressure.

Here, we report the preparation and characterization of silver nanoparticles capped with chitosan and their composites to develop films whose conductivity can be tuned with applied pressure. To the best of our knowledge, no work has been reported in this direction on silver - chitosan composites.

\section{Experimental Details}

2.1 Materials used

Silver Nitrate $\left(\mathrm{AgNO}_{3} 99.9 \%\right.$; $\left.\mathrm{AR}\right)$, Acetic acid glacial $\left(\mathrm{CH}_{3} \mathrm{COOH}, 99 \%\right.$; $\left.\mathrm{AR}\right)$, Sodium Borohydride $\left(\mathrm{NaBH}_{4}, 98.0 \%\right.$; AR), Chitosan $\left(\mathrm{C}_{6} \mathrm{H}_{11} \mathrm{O}_{4} \mathrm{~N}, 99.2 \%\right.$; Sigma Aldrich)-polymer and single distilled water is used in all synthesis part of the work.

\subsection{Synthesis of uncapped silver nanoparticles}

Mixture-1: $0.5 \mathrm{M}(4.2467 \mathrm{~g}) \mathrm{AgNO}_{3}$ added to $50 \mathrm{ml}$ distilled water.

Mixture-2: $0.5 \mathrm{M}(0.9457 \mathrm{~g}) \mathrm{NaBH}_{4}$ is added to $50 \mathrm{ml}$ distilled water. 
Mixture -2 added drop wise to Mixture-1 by maintaining constant time interval so that the particles obtained can be of uniform sized one. Thus the uncapped silver nanoparticles are prepared. These nanoparticles are extracted by repeated filtering and washing with distilled water. The powder is allowed to drying. The collected powder of nanoparticles was $2.6959 \mathrm{~g}$ which is of high yield. Finally the dried nanoparticles are grinded firmly and subjected to different methods of characterization.

\subsection{Synthesis of Silver nanoparticles capped with chitosan}

Mixture-1: $0.05 \mathrm{M}$ Chitosan $\left(\mathrm{C}_{6} \mathrm{H}_{11} \mathrm{O}_{4} \mathrm{~N}\right)$ added to $50 \mathrm{ml}$ distilled water taken in a clean beaker, $1 \mathrm{M}$ acetic acid $\left(\mathrm{CH}_{3} \mathrm{COOH}\right)$ added to it (Chitosan polymer dissolves in acetic acid). Name given to this mixture as Polymeric Solution (PS). $0.5 \mathrm{M}$ silver nitrate $\left(\mathrm{AgNO}_{3}\right)$ is added to PS and kept in ultrasonic bath for 10 min to attain homogeneity.

Mixture-2: $0.5 \mathrm{M}$ sodium borohydride $\left(\mathrm{NaBH}_{4}\right)$ added to $50 \mathrm{ml}$ distilled water in a clean beaker.

Mixture-2 added to Mixture-1 with constant stirring by maintaining constant time interval so that nanoparticles produced can be of uniform in size. In this case the solution slowly turned in to a brown color indicating the formation of silver nanoparticles. Further continuous adding solution turned in to dark green color indicating the nanoparticles formed are capped with chitosan. Hence silver nanoparticles capped with chitosan are prepared.

Nanoparticles were extracted by repeated washing with distilled water and are allowed to drying in petry-dish under table lamp. Since chitosan acts as capping agent the nanoparticles obtained are very smaller in size. The collected powder was $2.3616 \mathrm{~g}$ and here the yield is $87.6717 \mathrm{wt}$. \% which is reasonably high. The dried particles are grinded firmly and subjected to different characterizations.

Fig.1 shows photographic image of prepared silver nanoparticles in which the white coloured sample (left of Fig.1) contains uncapped silver nanoparticles. Another sample consists of silver nanoparticles capped with chitosan (right of Fig.1), in a common view it appears as black colour but its actual colour is dark greenish because of these silver nanoparticles capped with environmentally friendly green chitosan molecules.

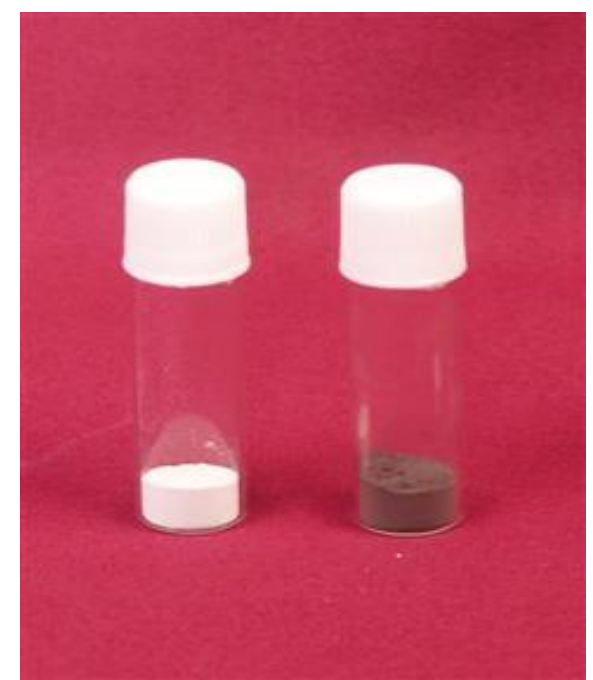

Fig.1: Photographs of prepared uncapped and capped silver nanoparticles under room light.

\subsection{Preparation of composite films}

Here, we report the preparation of films and electrical characterization of composites whose conductivity can be tuned with applied pressure.

Here in this work the combination of different weight percentage of Chitosan polymer and silver nanoparticles are taken (both capped and uncapped are taken separately) and made a composite film. To make composite film a known weigh percentage of chitosan polymer taken in test-tube containing $1.5 \mathrm{ml}$ distilled water. After the mixture becomes homogeneous condition, a known weight percentage of silver nanoparticles are added (capped and uncapped separately) to the mixture. Each mixture is homogenized using ultrasonic bath.

After the mixture becomes homogeneous, the mixture was grinded firmly till it become a paste. The paste was then transferred on glass-plate without spreading much to get thick film. It was allowed to dry for two days under table lamp. After drying the chitosan polymer acts as insulating matrix in the film. This is how the films of different compositions were made. During grinding it is found that the capped silver nanoparticles mixed well with chitosan, where as uncapped silver nanoparticles agglomerate together (in case of higher wt.\%) and do not get mixed with chitosan properly. As a result limited compositions were prepared with uncapped particles. 


\subsection{Characterization}

Freshly prepared solutions of both uncapped and capped silver nanoparticles were subjected to optical absorption measurements using HITACHI U-2800 UV-VIS spectrophotometer. For the confirmation of crystallographic phase of both uncapped and capped nanoparticles, X-ray diffraction was carried out using PHILIPS PW-1710 X-ray diffractometer To understand the surface morphology, powdered samples were subjected to SEM measurements using JEOL-JSM-6360 scanning electron microscope. Fourier transfer infrared (FTIR) spectroscopy measurements were done with NICOLET 5700 FTIR spectrometer under the transmission mode by loading nanoparticles in $\mathrm{KBr}$ pellets.

\subsection{Electrical conductivity measurements}

A simple set-up was designed and fabricated to measure electrical conductivity of films under applied pressure as shown in Fig.2.

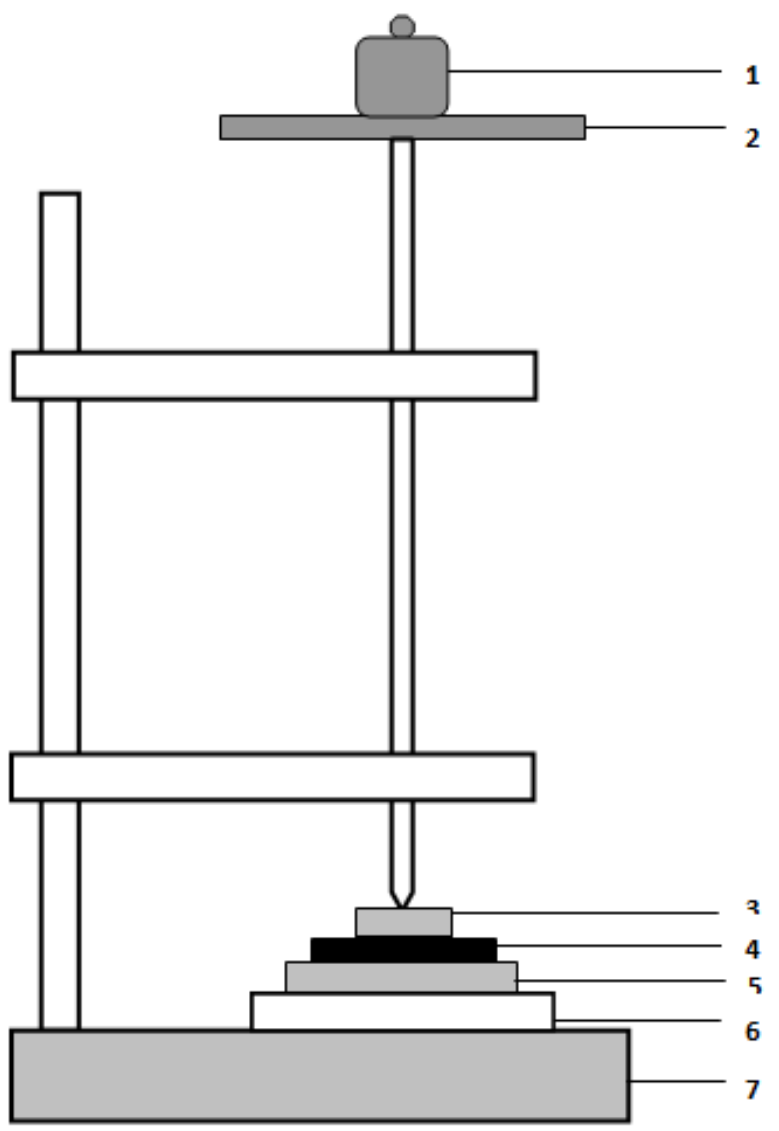

Fig.2: Experimental setup to study charge transport in composite films.

[ (1) Weight (2) Weight Holder (3) Upper Cu disc (UCD) (4) Composite film (5) Lower Cu plate (LCP) (6) Glass-plate

(7) Supporting stand ]

Different weights were put on the upper plate which was later converted to actual pressure. The film was placed between the upper and lower copper plates of the measurement setup. Average thickness of films was measured using Peacock dial gauge G Type (Ozaki, Japan), in terms of micrometer.

It consists of weights (used to apply the pressure), weight holder, Upper copper disc (UCD), Lower copper plate (LCP), the glass-plate is placed on the stand as shown in figure, above which the Lower copper plate (LCP) is placed which is the platform for measuring sample. The cross sectional area of Upper copper disc (UCD) is $86.93 \times 10^{-2} \mathrm{~cm}^{2}$. The composite film whose conductivity to be measured is placed in between UCD and LCP. The pressure is applied on UCD with the help of needle. The needle has the weight holder on its top to apply the pressure. The pressure is applied to the required amount by varying the weights from $0 \mathrm{~kg}$ to $2 \mathrm{~kg}$. For each pressure the Current - Voltage characteristics are measured across the composite films later the voltage is converted to electric field to compare different compositions.

The voltage is measured using KEITHLEY 617 programmable electrometer and current is measured by using KEITHLEY 197A autoranging microvoltmeter. A regulated power supply of range (0 to 30V) is used to apply the voltage. The series resister of resistance $199 \Omega$ is used to limit the current $(<15 \mathrm{~mA})$ through the film. 
The Halipot potentiometer of range 0 to $50 \mathrm{k} \Omega$ is used to vary the current in the circuit. For convenient, the top of the film is made positive with respect to bottom of the film for forward bias. The bottom tip of the weight holder is made to stand firmly on the top of the upper copper plate.

\section{Results and Discussion}

The absorption spectra in the UV-visible region were recorded at room temperature. The UV-visible spectra of uncapped and capped silver nanoparticles shown in Fig.3, the spectra gives information about the suspension of silver nanoparticles in the solutions prepared.

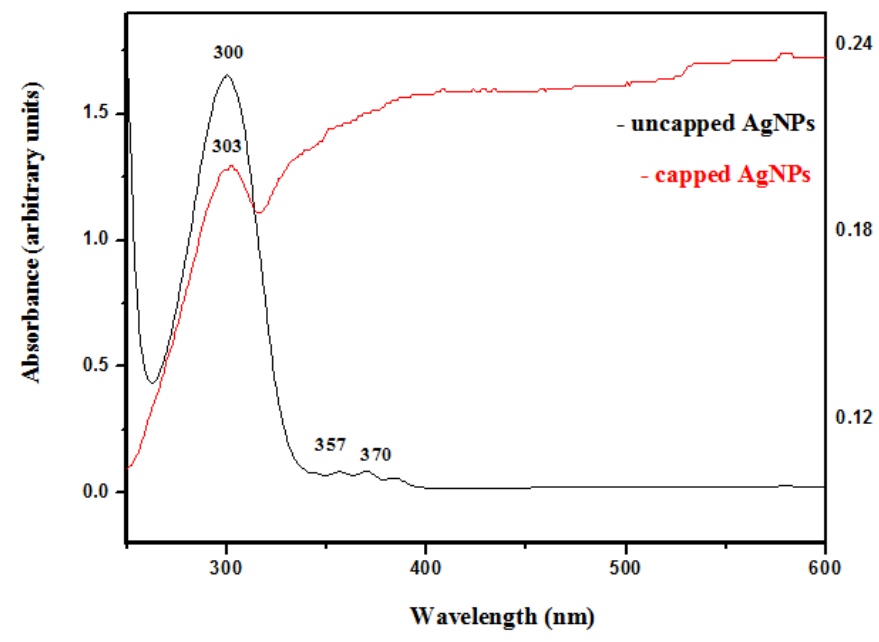

Fig.3 UV-visible spectra of uncapped and capped silver nanoparticles

Silver nanoparticles formed in the solution exhibiting an absorption peak around 300nm in case of uncapped silver nanoparticles and $303 \mathrm{~nm}$ in case of capped silver nanoparticles this is the characteristic of surface plasmon resonance (SPR) [30]. This Phenomenon is observed at the interface between metal and dielectric medium due to the interaction of metal electrons with incident light provided the size of the metal particles is above the critical value [31]. Numerous theoretical models and experimental results are published in an effort to understand the effect of particle size on the surface Plasmon resonance peak in nanomaterials, still the subject is not clearly resolved [32,33,34,35]

$\mathrm{X}$-ray diffraction patterns were recorded for both uncapped and capped silver nanoparticles samples in the range $2 \theta=10^{\circ}$ to $90^{\circ}$ using $\operatorname{Cuk} \alpha(\lambda=1.54056 \AA)$ radiation are shown in Fig.4.

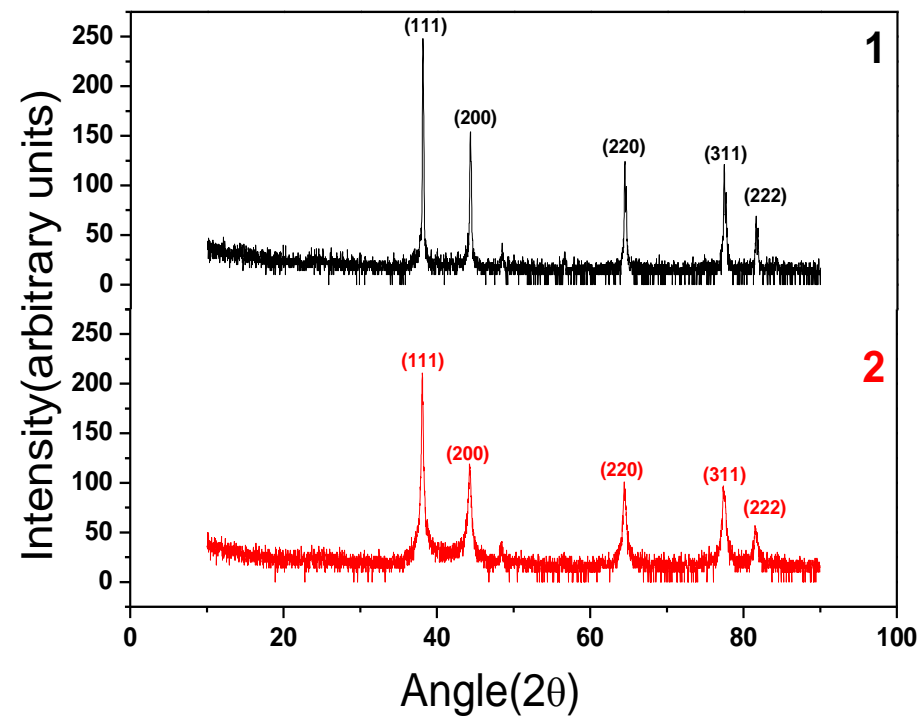

Fig.4. X-Ray Diffraction pattern for powder samples of uncapped (1) and capped (2) silver nanoparticles

By the observation of X-Ray pattern and calculations it is clear that, both uncapped and capped silver nanoparticles are having FCC structure and the peaks corresponding to (111), (200), (220), (311) and (222) 
Miller indices are clearly observed. The estimated parameters for this phase is $\mathbf{a}=\mathbf{4 . 0 7 1} \AA$ that is quite close to the lattice parameter of bulk silver $\mathbf{a}=\mathbf{4 . 0 8 6} \AA$. If we compare the sharpness of the peaks of these two spectra we observe that the peaks are very sharp in case of uncapped silver nanoparticles as compared to capped ones, indicating the size of uncapped silver nanoparticles is high and this is because of absence of chitosan molecules on the surface. In this case the particles merge together. Where as in the case of capped silver nanoparticles due to the presence of chitosan molecules on the surface, particles do not merge together and forms smaller size. Using Scherer formula size of nanoparticles was calculated. The average size (diameter) of uncapped nanoparticles is $34 \mathrm{~nm}$ and for capped nanoparticles it was $18 \mathrm{~nm}$ in size. These X-ray diffraction pattern obtained for prepared nanoparticles have given better experimental results with theoretical aspects studied in standard literatures.

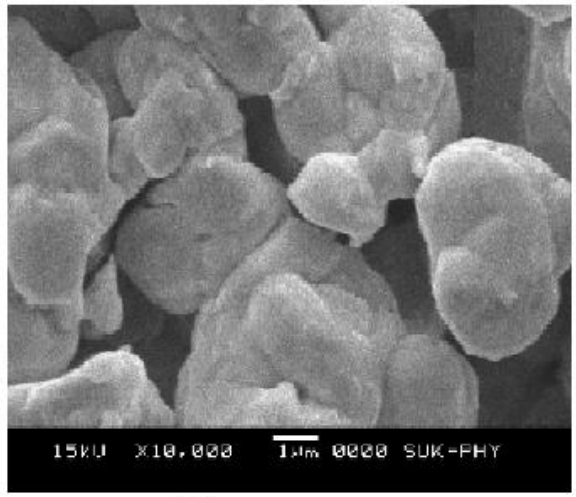

(1)

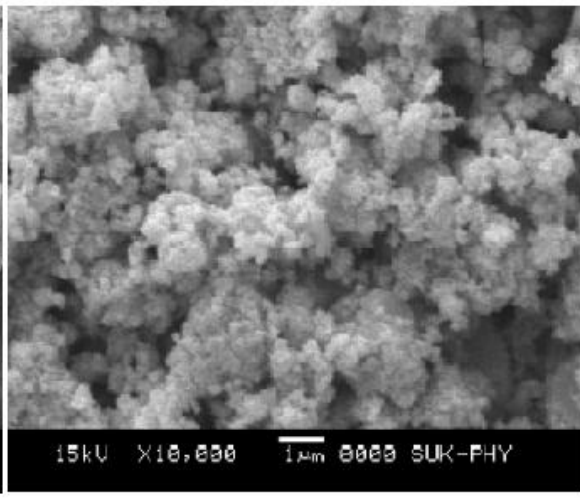

(2)

Fig.5 SEM images of uncapped (1) and capped (2) silver nanoparticles

Fig.5 shows SEM images of uncapped and capped silver nanoparticles. With the addition of reducing agents in the solvents, after formation of metallic nanoparticles they merge with adjacent nanoparticles and then form a microstructure (coagulation). And after repeated grinding the particles forms again a nanostructure with few particles aggregation in case of uncapped nanoparticles as shown in Fig.5 (1). By observing Fig.5 (2) directly we can say that the particles obtained are nano sized one, and are quite small compared to uncapped particles. Since chitosan molecules acts as a reducing agents in presence of aqueous sodium borohydride $\left(\mathrm{NaBH}_{4}\right)$, even after drying the chitosan molecules does not allow silver nanoparticles to get merge with each other. Thus in SEM image these particles appear as nano sized ones. Hence we confirm that the capped silver nanoparticles are much smaller in size compared to uncapped silver nanoparticles.

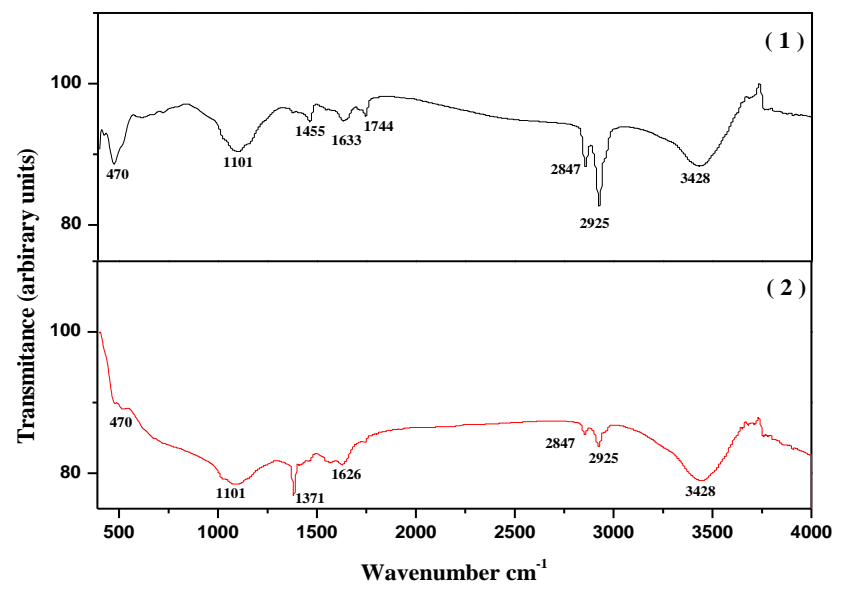

Fig.6 FTIR spectra of uncapped (1) and capped (2) silver nanoparticles.

Fig.6 displays the FTIR spectra of uncapped and capped silver nanoparticles. The powder of uncapped Silver nanoparticles shows stretching vibrations of $\mathrm{N}-\mathrm{O}, \mathrm{O}-\mathrm{H}$ and $\mathrm{N}-\mathrm{H}$ peaks at $1633 \mathrm{~cm}^{-1}, 2925 \mathrm{~cm}^{-1}$, and $3428 \mathrm{~cm}^{-1}$ respectively and bending vibrations of $\mathrm{N}-\mathrm{H}$ is at $1455 \mathrm{~cm}^{-1}$. That means still some nitrogen atoms are also bind on the surface of nanoparticles even after formation of nanoparticles. And FTIR spectrum for capped silver nanoparticles shows stretching vibrations of $\mathrm{C}-\mathrm{N}, \mathrm{N}=\mathrm{O}, \mathrm{C}=\mathrm{C}, \mathrm{O}-\mathrm{H}$ and $\mathrm{N}-\mathrm{H}$ peaks at $1101 \mathrm{~cm}^{-1}, 1371 \mathrm{~cm}^{-1}$, $1626 \mathrm{~cm}^{-1}, 2925 \mathrm{~cm}^{-1}$ and $3428 \mathrm{~cm}^{-1}$ respectively. It indicates that many of chitosan molecules are present on the 
surface of Silver nanoparticles even after drying. Due to presence of these chitosan molecules on the surface of silver nanoparticles, the particles will not get merge together. They form much smaller in size comparing to uncapped ones due to this reason only.

If we observe carefully in the region $1371 \mathrm{~cm}^{-1}$ there is a presence of a strong and extra peak indicating stretching vibration of $\mathrm{N}=\mathrm{O}$. This shows chitosan is the best capping agent for silver nanoparticles. Hence the FTIR spectroscopy given the better results to confirm about presence of chitosan molecules on the surface of silver nanoparticles with theoretical considerations.

To study the charge transport in uncapped and capped silver nanoparticles electrical conductivity measurements were carried using KEITHLEY meters. Observations made by taking I-V characteristics for four different composite films consisting $90 \mathrm{wt} . \%, 70 \mathrm{wt} . \%, 60 \mathrm{wt} . \%, 50 \mathrm{wt} . \%$ of capped silver nanoparticles with 10 wt. $\%, 30$ wt. $\%, 40 \mathrm{wt} . \%, 50 \mathrm{wt} . \%$ of chitosan polymer respectively. I-V characteristics of these compositions are shown in Fig.7 The Fig.7 is the plot for I-V characteristics of capped nanoparticles in logarithmic form.

Here we can observe in Fig.7 (a) the film consisting highest wt.\% of capped silver nanoparticles shows high current. As wt.\% of capped silver nanoparticles decreases in the film, the curve shows lesser current. The same experiment was carried for the composite films consisting different wt. $\%$ of uncapped silver nanoparticles. In this case we could able to prepare the composite films consisting of 70,60 , and $50 \mathrm{wt} \%$ of uncapped silver nanoparticles and 30, 40 and $50 \mathrm{wt} \%$ of chitosan polymer. Due to coagulation of more number of uncapped silver nanoparticles in the center of the composite film, the composite film consisting $90 \mathrm{wt} . \%$ of uncapped silver nanoparticles and $10 \mathrm{wt} . \%$ of chitosan polymer was not taken for electrical measurements. And also this forms inhomogeneity. I-V characteristics of these compositions are shown in Fig.7 (b).

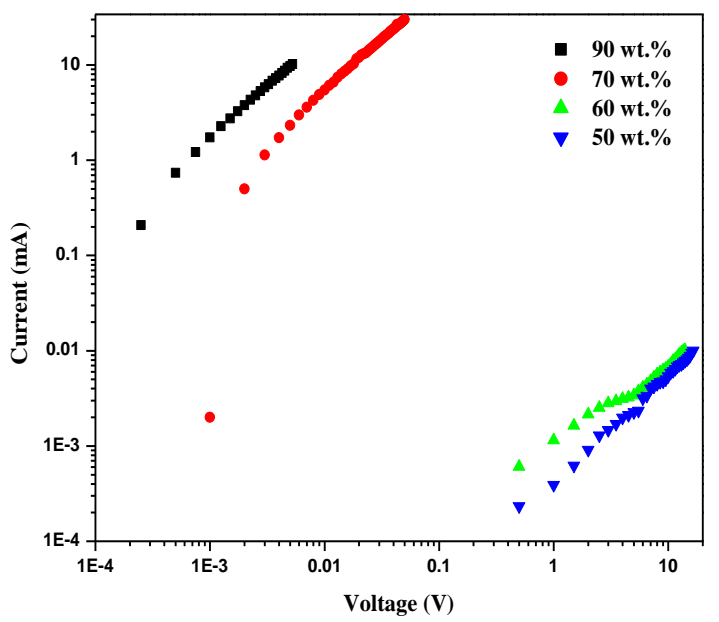

Fig.7 (a) Plot of current vs. voltage for the composite films consisting of different wt.\% capped silver nanoparticles.

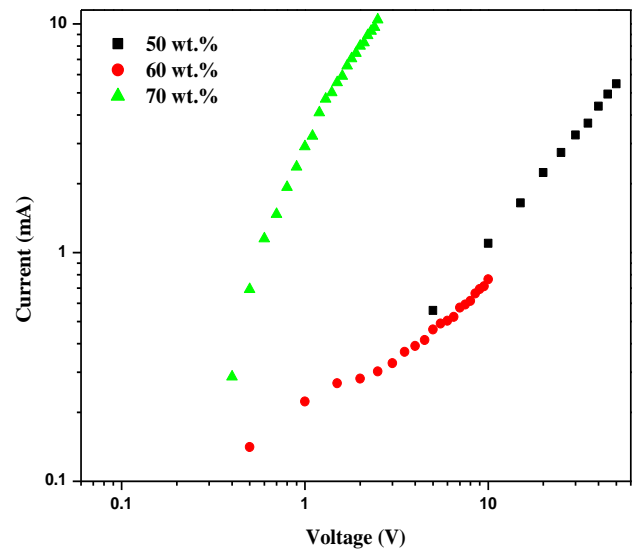

Fig.7 (b) Plot of current vs. voltage for the composite films consisting of different wt.\% of uncapped silver nanoparticles. 
Fig.7 (b) is the plot of I-V characteristics for uncapped nanoparticles in logarithmic form. Here also the same thing we can observe as in I-V characteristics of composite films consisting capped silver nanoparticles. In Fig.7 (b) the very first curve shows high current due to the presence of rich uncapped silver nanoparticles in film. As we go for lower weight percentage of uncapped silver nanoparticles in the composite film, the curves shows less current.

Using these I-V characteristics of uncapped and capped silver nanoparticles we further carried the observations like the plots for conductivity as a function of weight percentages of silver nanoparticles in composite films, conductivity as a function of applied pressure on the composite films and finally comparison between two films was identified for higher conductivity.

For the composite film consists 90 wt.\% of capped silver nanoparticles and $10 \mathrm{wt} \%$ of chitosan polymer conductivity occurred when $2 \mathrm{~kg}$ load applied is $3.806 \times 10^{-2} \mathrm{mhos}_{\mathrm{cm}} \mathrm{cm}^{-1}$ for same load if we go for low weight percentage of capped silver nanoparticles in composite film, we find still lower conductivity i.e., for 70

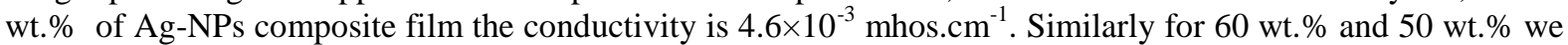
get $7.4 \times 10^{-9}$ mhos.cm $\mathrm{cm}^{-1}$ and $7.301 \times 10^{-9}$ mhos.cm ${ }^{-1}$ respectively indicating that there will be a decrease in conductivity as decrease in weight percentage of capped Ag-NPs in composite film.

Fig.8 (a) shows there is an increase in conductivity as weight percentage of increases in the composite film as discussed above. It is also observed that, there will be occurrence of decrease in conductivity as we go for lower load (pressure applied). If we plot the graph of conductivity vs. weight percentage of uncapped silver nanoparticles it is observed that there is high conductivity for higher weight percentage of uncapped silver nanoparticles in composite film and as we go for lower weight percentage of silver nanoparticles in composite films. Here below Fig. 8 (b) shows the plot for conductivity vs. weight percentage of silver nanoparticles in composite film.

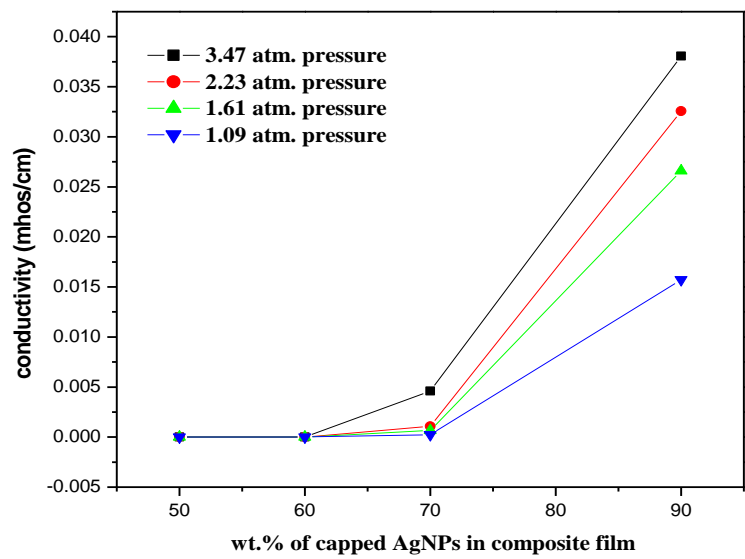

Fig.8 (a) conductivity vs. wt.\% of capped Ag NPs in composite film.

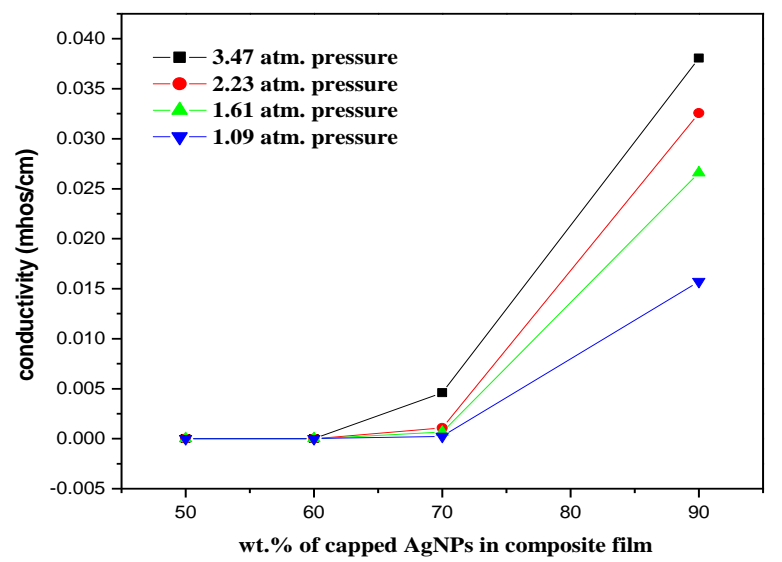

Fig.8 (b) conductivity vs. wt.\% of uncapped Ag NPs in composite film. 
In the Fig.8 (b) we can also observe that there is increase in conductivity as pressure applied increases by considering plots we planned to plot the 'graphs for conductivity as a function of pressure applied in both cases of uncapped and capped silver nanoparticles.

Fig.9 (a) shows the plot for conductivity against applied pressure on the composite film consisting of capped silver nanoparticles.

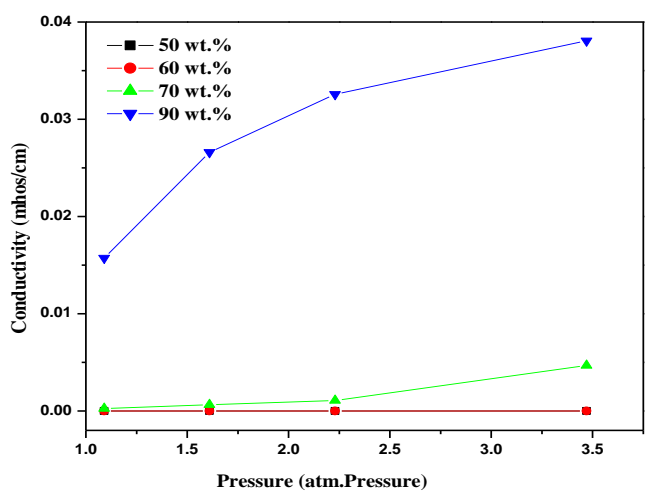

Fig.9 (a) conductivity vs. applied pressure [capped AgNPs]

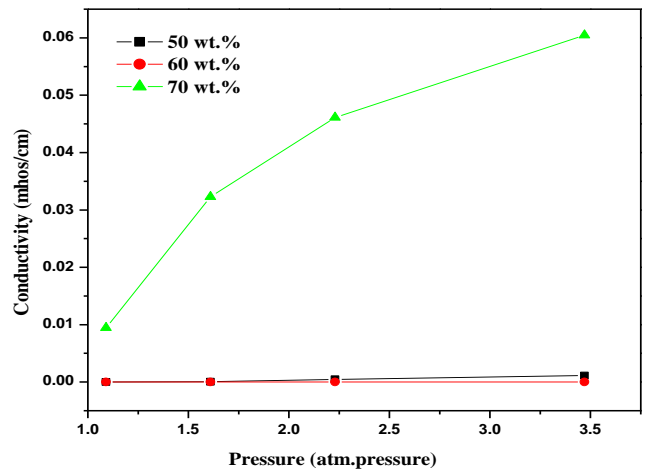

Fig.9 (b) conductivity vs. applied pressure [uncapped AgNPs]

Here we observe the pressure dependent conductivity in case of capped silver nanoparticles, conductivity decreases with respect to applied pressure. The same results obtained in case of the composite films containing uncapped silver nanoparticles. The Fig.9 (b) shows the plot for conductivity vs. applied pressure. The top curve corresponding to the composite film consisting $70 \mathrm{wt} \%$ of uncapped silver nanoparticles showing high conductivity. And conductivity decreases as concentration of uncapped silver nanoparticles decreases in composite film. With considering above results we plotted the graph of conductivity against percentage of nanoparticles in composite film.

The Fig.10 gives the comparison between two composite films consisting capped and uncapped silver nanoparticles separately.

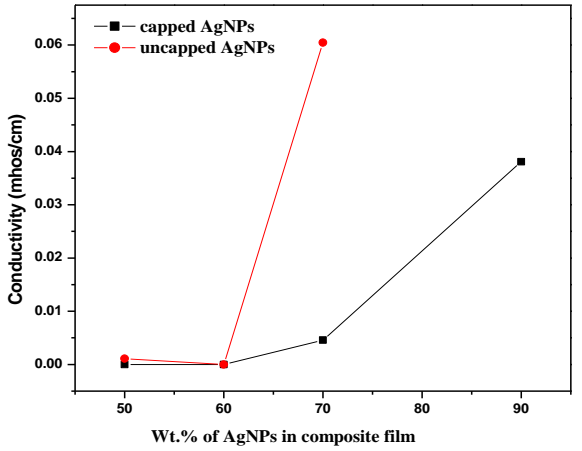

Fig.10. Conductivity vs. Wt.\% of AgNPs in composite film 
This curve of composite films which consisting of uncapped silver nanoparticles shows high conductivity than that of capped silver nanoparticles. Another point we must mention that due to accumulation of chitosan molecules on either surfaces of composite film decrease in conductivity occurs. Chitosan molecules resist the flow of charges in composite film and again if we apply some pressure on composite film, then there is a reasonably more flow of charges occurs. As a result conductivity increases. Thus we can observe that increase in conductivity with respect to pressure. By all above results we conclude that these composites can be used in simple low-cost pressure sensors.

\section{Conclusions}

Nanocomposites of silver and chitosan have been synthesized that display interesting and technologically useful functional properties. Several nanocomposites have already been produced that are currently being used in commercial applications and there is great promise that many more technologically useful systems will be available in the future. Processing methods have been developed that allows very precise control of the microstructure that in turn allows for sensitive tuning of these properties. In the present work we aimed to produce composite materials of silver nanoparticles-chitosan that can have superior electronic properties.

The synthetic scheme which we have followed is a simple chemical reduction method; the particles prepared by this method are stable. The Chitosan-Silver composite nanoparticles generated in the present method are of uniform and narrow in size less than $20 \mathrm{~nm}$. Our intension to prepare these capped and uncapped nanoparticles was to find difference in charge transport, thus we can find difference in conductivity. The FTIR results suggest chitosan is convenient capping agent for Silver nanoparticles. The synthetic method which we developed in present study might be useful for further development of green nanoparticles synthesis in other situations.

It is found in the present work that the chitosan capped silver nanoparticles have better mixing ability with chitosan. Current - voltage curves studied for composites of both capped and uncapped silver nanoparticles with chitosan to understand the electronic charge transport. Thus capped nanoparticles exhibits superior electrical properties and they can find potential applications in developing low-cost pressure sensors.

\section{References}

He H, Zhu J, Tao N J, Nagahara L A, Amlani I and Tsui R, J. Am. Chem. Soc. 123, 7730 (2001)

Stucky G D, Nature. 410, 885 (2001)

Jager E, Ingalis O, Lundstrom L, Science. 288, 2335 (2000)

Bashyam R, Zelenay P, Nature. 443, 63 (2006)

Sunoyama H, Sakurai H, Negishi Y and Tsuguda T, J. Am. Chem. Soc. 109, 692 (2005)

Zhang J, Xu S and Kumacheva E, J. Am. Chem. Soc., 126, 7908 (2004)

Calo V, Nacci A, Monopoli A, Fornaro A, Sabbatini L, Cioffi N and Ditaranto N Organometallics. 22, 5154 (2004)

Vincent T and Guibal E, Langmuir. 19, 8475 (2003)

Sih B S and Wolf O M, Chem. Commun. 2005, 3375 (2005)

Novak P J and Feldheim L D, J. Am. Chem. Soc.122, 3979 (2000)

Atay T, Song J-H and Nurmikko V A, Nano Lett. 4, 1627 (2004)

Kobayashi S, Toshitsugu k and Shin-ichiro S, J. Am.Chem. Soc. 118, 13113 (1996)

Shin Y, Yoo D I and Jang J, J. Appl. Polym. Sci. 74, 2911 (1999)

Shigehiro H, Yasuo K, Shuntaro K, Testsuo T, Hisaya T, Kakuko M and Takeshi Y, Biochem. Syst. Ecol.19, 379 (1991)

Domare A and Roberts G A F, Advances in Chitin Science ed J Andre, National Taiwan Ocean University, Keelung: (1998)

Dhanikula A B and Panchangnula R, AAPS J. 6, 1 (2004)

Payne F G and Raghavan S R, Soft Matter. 3, 1 (2007)

Roy K, Mao H Q, Huang S K and Leong K W, Nature Med. 5, 387 (1999)

Bartkowiak A and Hunkeler D, Chem. Mater. 11, 2486 (1999)

Suzuki T, Mizushima Y, Umeda T and Ryo O, J. Biosci. Bioeng. 88, 194 (1999)

de Leeuw D. Plastic electronics. Phys. World. (1999)

Chiang CK, Fincher CR, Jr, Park YW, Heeger AJ, Shirakawa H, Louis EJ, Gau SC, MacDiarmid AG. Phys. Rev. Lett. 39,1098 (1977)

Kaner RB, MacDiarmid AG. Plastic that conduct electricity. Sci Amer 1988; 60, 258 (1988)

Stejskal J, Gilbert RG. Polyaniline: Preparation of a conducting polymer. Pure Appl. Chem. 74, 857 (2002)

Oyama N, Tatsuma T, Sato T, Sotomura T. Dimercaptan-polyaniline composite electrodes for lithium batteries with high energy density. Nature. 373, 598 (1995)

Rodriguez J, Grande HJ, Otero TF. Handbook of organic conductive molecules and polymers. Nalwa H.S., Ed.; John Wiley \& Sons Ltd. 1997, Vol. 2.

Balberg I. Excluded-volume explanation of Archie's law. Phys Rev B. 33, 3618 (1986)

Balberg I, Binenbaum N, Wagner N. Percolation thresholds in the three- dimensional sticks system. Phys Rev Lett. 52,1465 (1984)

Balberg I, Binenbaum N. Invariant properties of the percolation thresholds in the soft-core-hard-core transition. Phys Rev A. 35, 5174 (1987)

K.L. Kelly, E. Cicronado, L.L. Zhao, G.C.Schatz,; J.Phys. Chem. B. 107,668 (2003)

G.W. Arnold, J.A. Borders, J. Appl. Phys. 48, 1488 (1997)

B.N.J. Persson, A. Liebsch, Phys. Rev. B. 28, 4247 (1983)

S. Fedrigo, W. Harbich, J. Buttet, Phys. Rev. B. 47, 10706 (1993)

H. Hovel, S.Fritz, A. Hilger, U. Kreibig, Phys. Rev. B. 48, 18178 (1993)

W Cai, H. Hofmeister, M. Dubiel, Eur. Phys. J. D 13, 245 (2001) 